\title{
Neurologica
}

\section{Comparison of ventricular drain location and infusion test in} hydrocephalus

\begin{tabular}{|r|l|}
\hline Journal: & Acta Neurologica Scandinavica \\
\hline Manuscript ID & ANE-O-11-15-552.R2 \\
\hline Manuscript Type: & Original Article \\
\hline Therapy Areas: & Hydrocephalus, Spinal fluid and hydrocephalus \\
\hline Keywords: & Infusion test, ventriculoperitoneal shunts, Hydrocephalus \\
\hline \multicolumn{2}{|l}{} \\
\hline
\end{tabular}

SCHOLARONE ${ }^{1 m}$

Manuscripts 


\section{Comparison of ventricular drain location} and infusion test in hydrocephalus

Rohitashwa Sinha, ${ }^{1}$ BMBS

James AD Morgan, ${ }^{1 *}$ MBBChir

James R Wawrzynski, ${ }^{1}{ }^{*}$ MBBChir

Zofia Czosnyka, $\mathrm{PhD}^{1}$

Magdalena Kasprowicz, ${ }^{2} \mathrm{PhD}$

Marek Czosnyka, ${ }^{1}$ Prof., $\mathrm{PhD}$

Matthew Garnett, ${ }^{1}$

Peter JA Hutchinson, ${ }^{1}$ Prof, PhD

John D Pickard, ${ }^{1}$ Prof, FMedSci

Stephen J Price, ${ }^{1} \mathrm{PhD}$

1. Academic Department of Neurosurgery, Addenbrooke's Hospital, A Block, Box 166, Hills Road, Cambridge, England, CB2 0QQ

2. Department of Biomedical Engineering, Wroclaw University of Technology, Wybrzeze Wyspianskiego 27, 50-370 Wroclaw, Poland

\section{Corresponding author:}

Marek Czosnyka

Neurosurgical Unit,

Box 167 Department of Clinical Neuroscience

Cambridge cb20qq, UK

E-mail: mc141@medschl.cam.ac.uk

* These authors contributed equally to this work

Keywords: Infusion tests, ventriculoperitoneal shunts, hydrocephalus

Running head: Validating shunt infusion tests with CT imaging

Manuscript word count excluding abstract, references, tables and figures: 4331 


\begin{abstract}
Objectives: Suspected cerebrospinal fluid shunt (CSF) dysfunction in hydrocephalic patients poses a diagnostic uncertainty. The clinical picture can be nonspecific and CT imaging alone is not always pathognomic. Infusion tests are an increasingly used investigation for real-time hydrodynamic assessment of shunt patency. We report correlation between infusion test results with quality of ventricular drain placement on CT image in large retrospective group of hydrocephalic patients.

Materials \& methods: 306 infusion test results performed in 200 patients were correlated with 306 corresponding CT head scans. Nominal logistic regression was used to correlate shunt catheter position on imaging to patency of ventricular drain as determined by infusion tests.

Results: Infusion test results of shunt patency are statistically congruent with the analysis of shunt catheter position on CT head scans. Catheter tips completely surrounded by either parenchyma or CSF on CT imaging, are strongly associated with evidence of occlusion or patency from infusion tests, respectively $\left(\chi^{2}=51.68\right.$, $\mathrm{p}<0.0001, \mathrm{n}=306$ and $\chi^{2}=31.04, \mathrm{p}<0.0001, \mathrm{n}=306$ ).
\end{abstract}

Conclusions: The most important anatomical factor for shunt patency is the catheter tip being completely surrounded by CSF. Infusion tests provide functional and reliable assessment of shunt patency in vivo and is strongly correlated with the position of the ventricular catheter on CT imaging.

Keywords: Infusion test, ventriculoperitoneal shunts, hydrocephalus 


\section{Introduction}

Only modest progress has been made in preventing ventriculo-peritoneal shunt failure for several decades ${ }^{1}$. Shunt revision is still a common neurosurgical operation, posing significant risks to patients and a heavy financial burden on healthcare services ${ }^{2}$. Recent long-term studies have quoted shunt failure rates requiring revision surgery of $23 \%^{3}$ and $46 \%{ }^{4}$ over 8 years. Mechanical dysfunction accounts for $74 \%$ of such revisions ${ }^{3}$ with occlusion of the proximal catheter being the most common site for obstruction $^{5}$.

Electron microscopic and histological evidence from blocked ventricular and peritoneal catheters show that the cause of occlusion is often debris from choroid plexus and ependyma ${ }^{6,7}$. Therefore it has been suggested that the optimal position for the tip of the ventricular catheter ought to be in the ipsilateral frontal horn anterior to the foramen of Monro ${ }^{8-10}$, since the choroid plexus is absent from this part of the ventricle.

Since Albright and colleagues quoted only $55 \%$ of the frontally placed catheters were

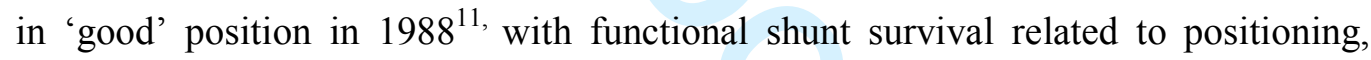
more recent studies still quote high risk of sub-optimally placed frontal catheters; up to $56 \%{ }^{12}$. This is in part likely due to the fact that the freehand ventriculostomy has traditionally been undertaken as a 'blind' procedure, relying on surface anatomical landmarks to guide insertion. This notion is supported by studies of other procedures requiring 'blind' ventriculostomy such as external ventricular drain insertion which also show high incidence of suboptimal ventricular catheter positioning ${ }^{13,14}$. Unsurprisingly, this has led to a shift towards using image guidance or other tools to aid in improving the accuracy of ventricular cannulation ${ }^{12,15}$.

Many studies investigating shunt dysfunction have used the clinical assessment of patients, the decision to revise a shunt and Computed Tomography (CT) imaging to assess catheter position and changes in ventricular size as surrogate markers to infer the patency of shunts ${ }^{1,3,4,7}$. Increasingly in clinical practice, infusion tests are being undertaken as a means to be objectively measure the flow dynamics of fluid within shunts in vivo ${ }^{16-19}$, especially when the diagnosis of shunt dysfunction is not clearly 
evident from the clinical presentation and changes in ventricular size on CT alone, which may never happen in normal pressure hydrocephalus (NPH). When infusion test technique may be seen as little bit outdated, and new theories for development of hydrocephalus are proposed ${ }^{20}$, using of infusion test to scrutinize shunt performance in vivo seems to be clinically well-funded. ${ }^{21}$

In this study, we investigate the relationship between ventricular catheter position on CT scans using an objective classification and their effect on patency of shunt's system ventricular drain as assessed by infusion tests. The study was conducted retrospectively in prospective patients with symptoms of shunt dysfunction where the diagnosis was ambiguous (i.e. patients were not just revised based on imaging and clinical symptoms, but referred by consultant neurosurgeon in charge to Hydrocephalus Clinic, to conduct infusion test).

\section{Matherial and Methods}

A retrospective, non-controlled, observational prospective cohort was studied. All data used was derived from clinical records of patients at Addenbrookes Hospital, Cambridge University Hospitals NHS Trust. Due to the use of retrospective historical records and no active patient involvement during the data collection, patient consent for the use of data in research was taken pre-emptively by Addenbrooke's Hospital, Cambridge University Hospitals NHS Trust as part of routine audit protocol. Patients who had an infusion test on the basis of clinical symptoms of ventriculo-peritoneal shunt dysfunction between 2007 and 2011 were identified. The results of their infusion tests were analysed and classified according to the state of ventricular drain either obstructed or patent (see descriptions below).

To correlate these physiological evaluations of shunt patency with the anatomical position of the proximal catheter tips, we analysed the CT head scans for the same patients within this period using a new classification system. We excluded scans with a greater than three month interval between scan and infusion test, and any patients that had revision operations within this time to ensure that the same shunt proximal catheter was being assessed physiologically by infusion test and radiologically by CT 
scan. Data from both the physiological and radiological assessments were correlated statistically.

\section{Infusion test and its analysis}

The infusion test is a technique, modified from the "Constant-Infusion Manometric Test" described by Katzman and Hussey ${ }^{22}$. It involves the insertion of two $25 \mathrm{G}$ butterfly needles into the subcutaneous shunt reservoir, which is connected with the intra-ventricular catheter. Via one needle, saline is infused at a constant rate, whilst the other records pressure changes (Fig 1 upper panel). During the infusion, the computer calculates and presents mean pressure and pulse amplitude [with time along the $\mathrm{x}$ axis (see Figure 1 lower panel)]. Pressure increases slowly, as a compliance of CSF space is substantial and pulse amplitude of pressure waveform usually increases proportionally to pressure. The resistance to CSF outflow can be calculated using simple arithmetic as the difference between the value of the plateau pressure during infusion and the resting pressure divided by the infusion rate. Repeatability of calculation of the resistance to CSF outflow has been investigated before ${ }^{23}$. However, the precise measurement of the final pressure plateau is not possible when strong vasogenic waves arise or an excessive elevation of the pressure above the safe limit of $40 \mathrm{~mm} \mathrm{Hg}$ is recorded. Computerized analysis produces results even in difficult cases when the infusion is terminated prematurely. The algorithm utilizes time series analysis for volume-pressure curve retrieval, the least-mean-square model fitting and an examination of the relationship between the pulse amplitude and the mean CSF pressure. The pressure-volume curve is investigated.

Infusion test does not need special technical assistance. Recording lasts on average 30 minutes. Risk of infection is limited to less than $1 \%$ according to internal audit. It is helpful if it is supported by specialized software like computer program (Intensive Care Monitor (ICM+), Cambridge Enterprise Limited ${ }^{24}$ which processes the recorded data to provide graphical waveforms of intracranial pressure, heart rate and pulse amplitude derived from intracranial pressure fluctuations whilst saline is being infused. Infusion tests have been shown to provide an objective evaluation of shunt function in vivo ${ }^{16-18}$ with satisfactory (90\%) positive predictive power ${ }^{16,21}$.

All infusion tests performed in patients with suspected non-functioning ventriculoperitoneal shunts at Addenbrooke's Hospital between 2007 and 2011 were assessed. 499 infusion tests met the inclusion criteria above. 
With these objective measurements, infusion tests can distinguish between shunts that are:

1. functional,

2. high opening pressure but not indicative of obstruction, i.e. calculated outflow resistance in agreement with nominal resistance of opened shunt, indicating increased abdominal pressure,

3. obstructed - distal ${ }^{21,25}$,

4. obstructed - proximal.

For the purposes of this study we classified groups 1,2 and 3 above as shunts with patent ventricular drain and group 4 as obstructed.

Each group has a distinct pattern on the pressure waveform produced during the infusion test. In the following two figures the differences between functional and a proximally obstructed shunt are demonstrated.

In Figure 2, three essential features consistent with correct functioning ventricular end of shunt system are illustrated. Firstly, the normal baseline intra-cranial pressure (ICP) increases slowly to the value characterizing the resistance to CSF outflow. Secondly the heart rate is detectable throughout the infusion phase and lastly the pulse amplitude (AMP) is recordable, both features demonstrating patency of the ventricular drain. Both pulse amplitude (AMP) and heart rate (HR) are estimated using adaptive heart rate tracking spectral analysis method. 1024 point discrete power spectrum is calculated using 10 second intervals of sampled $(100 \mathrm{~Hz})$ ICP signal. Spectrum peak associated with a heart rate (measured using distal plethysmographic method) is identified manually to start the algorithm. Then it is tracked from one window to another during the course of the study. AMP is calculated from a power of first harmonic of spectral peak. During this procedure much of the fluid goes into the brain depending on CSF resistance to outflow possibly against sagittal sinus pressure, and further- down the shunt after its opening pressure is exceeded.

Figure 3 demonstrates several key differences in an obstruction of ventricular drain of shunt system. There is no pulse waveform of ICP recorded at baseline. Primarily the 
ICP immediately rises to the pressure level (shunt opening pressure plus abdominal pressure level) above which the valve opens distally. When the distal flow is obstructed during the test (for example, by pressing Siphon Control Device) the pressure rises further and without any delay, demonstrating an isolated circuit not in continuation with the ventricular space. The estimated heart rate is erratic and inconsistent and the pulse amplitude remains almost undetectable, which further shows a lack of continuity between the shunt and ventricular space. Opposite to previous scenario (Fig. 2), during this procedure all infused fluid presumably goes down the shunt into the abdomen influenced by the valve resistance, opening pressure, length of catheter and abdominal pressure.

For distal obstruction, plateau pressure reached during the study (Fig. 2) exceeds the level evaluated as: valve's opening pressure plus infusion rate times hydrodynamic resistance of the opened valve plus certain credit for abdominal pressure $(5 \mathrm{~mm} \mathrm{Hg}$ in non-obese patients) ${ }^{21,25}$.

\section{CT Analysis}

The electronic records of the patients who had undergone the 499 infusion tests between 2007 and 2011 were screened to identify those with a CT Head scan within three months of the test and no surgery between the test and scan. Although ventricle size can change rapidly, the positioning of ventricular catheters tends to stay static in serial imaging, hence this large timeframe was chosen to allow for greater data capture whilst minimising bias from different ventricular catheters. ${ }^{26}$

In total $306 \mathrm{CT}$ head scans of 200 patients were analysed. No coronal or 3D reconstructions were used. This was to simplify the analysis and ensure ease of transferability so that other clinicians could assess their scans in the same way. The slice thickness was $5 \mathrm{~mm}$ per axial cut.

Some patients had repeated clinical presentations where shunt dysfunction was considered leading to repeat infusion tests and CT scans. In those cases infusion tests and scans classification was interpreted as independent.

For this CT analysis a new classification tool was designed by the investigators to define the anatomical position of the proximal catheter tip. The goal was to create a highly specific, reproducible, easy-to-use and objective measure of location. This was 
achieved via the creation and use of simple categories with mutually exclusive options, which in combination can exhaustively express all possible tip positions and determines relevant anatomical relationships to aid in the comparison of shunt tip positions. These are outlined below:

1. Entry - Frontal OR Parietal

2. Entry - Left OR Right

3. Tip Position Relative to Entry - Ipsilateral OR Midline OR Contralateral

4. Tip Position Relative to Foramen of Monro within CSF space - Anterior OR Posterior OR Not in ventricular space

5. Tip Surroundings - Completely surrounded by CSF OR Completely surrounded Parenchyma OR Neither completely surrounded by CSF nor parenchyma

6. Presence of "Collapsed Ventricles" - Yes OR No

The classification of tip position relative to surroundings assesses the immediate environment of the drainage holes at the distance of $1.5 \mathrm{~cm}$ of the ventricular catheter. The word "completely" clearly divides those shunts in an optimal position (CSF) from those in a definitively suboptimal position (parenchyma) and groups those in between. This third group refers to those catheter tips which would be lying partially against a ventricular wall, brain parenchyma, septum pellucidum or lying within the foramen of Monro for example, whilst being partially surrounded by CSF.

For a catheter tip to be classified as anterior to the foramen of Monro, it had to lie within the ventricular space anterior to a coronal plane in line with the foramen. We also made an additional record in the cases where the catheter tip was seen to pass into the Foramen of Monro, rather than remaining anterior to it.

A midline classification refers to those shunt tips which lie along midline structures such as septum pellucidum or the third ventricle. A classification of contralateral position means that the tip has passed across the midline structures. 'Collapsed ventricles' refers to the radiographic evidence of narrowed or slit ventricles.

Shunt tip position on CT is a surrogate marker for the actual tip, as the CT axial plane slices are between $5-10 \mathrm{~mm}$ generating a small margin of error. Additionally, the 
drainage holes in the catheter are not limited to the tip and extend proximally for between $15 \mathrm{~mm}$ to $30 \mathrm{~mm}$.

General scheme of tip's position classification and relevant examples are presented in Fig. 4.

\section{Reliability}

$30 \mathrm{CT}$ scans from our sample were selected using a random number generator and were then independently re-analysed by each of the first three authors using the classification system described above. These results were statistically compared to assess for inter-observer variability and thus assess the overall reliability of our method.

\section{Statistical Analysis}

Nominal logistical models were used to analyse the effect of the explanatory variables (CT classification descriptors) and their biologically relevant two-way interactions on the dependent variable (shunt patency or proximal occlusion detected during infusion test), and were subject to step-wise removal starting with the least significant interaction term. All rejected terms were individually reintroduced to the reduced model to confirm they did not improve final model performance. Analyses were performed in JMP 7 (SAS Institute, Marlow, UK).

\section{Results}

306 infusion studies of in vivo shunts and their corresponding CT head scans were analysed. The age range of the patients was $1-88$ years with a mean age of 42 and the gender ratio was 2:3 (male:female). The median time between scan and infusion test was 1 day (interquartile range: 0 days -9 days). Table 1 details the results of the analysed CT head scans as categorised by proximal shunt patency determined by infusion test.

Out of 306 tests, 57 showed pattern of proximal blockage. The proximal patency of the shunt on infusion test was significantly associated with the matter it was surrounded by on CT scan (Table 2). 
If the shunt was completely surrounded by brain parenchyma on CT scan, it had the

highest likelihood of obtaining an infusion test result consistent with occlusion (33/62 occluded $\left.=53.22 \% ; \chi^{2}=51.68, \mathrm{p}<0.0001, \mathrm{n}=306\right)$. If the shunt was completely surrounded by CSF it had the least likelihood of becoming occluded (2/92 occluded $=$

$\left.2.17 \%, \chi^{2}=31.04, p<0.0001\right)$ as shown in Fig. 5. Shunts that were partially surrounded by both brain and CSF were significantly worse than being surrounded by CSF and significantly better than being surrounded by brain; with a highly significant difference between the two extreme groups (parenchyma versus CSF, $\mathrm{p}<0.000001$ ). None of the remaining categories for classifying catheter tip position on CT were significantly associated with proximal patency or proximal blockage detected during infusion test (Table 2). Of the 249 infusion tests with results of hydrodynamic patency, 29 had their catheter tips surrounded by parenchyma (12\%).

Our model provided no evidence for frontal or parietal approach shunts to be significantly different in likelihood of patency, when the effect size from surrounding matter was removed. In addition, for the frontal shunts with catheter tips in the frontal horn of the lateral ventricle, further data was collected to classify the position within the ventricle (anterior to foramen of Monro, through foramen of Monro and abutting septum pellucidum). The location of these catheter tips was also not significantly associated with patency.

Intraobserver agreement has been tested additionally and was assessed as high (CHI2 $=2.65 ; \mathrm{p}=0.85$ ).

Although this was not a formal subject of the current study, but unconfirmed incidence of distal blockage was 72 cases (23\%). Incidence of cases with patent shunt (normal resistance to CSF outflow) but presumably increased abdominal pressure was 25 (8\%). Totally, almost $50 \%$ of infusion tests suggested shunt malfunction and remaining - no evidence of malfunction.

\section{Discussion}

In approximately $19 \%$ of infusion tests in patients with clinical symptoms of problems potentially associated with shunt malfunction, proximal occlusion can be seen. Infusion test results of proximal patency were very strongly associated to the position of the ventricular catheter tip on CT head imaging. Patients proven to have 
proximally blocked shunts by infusion test were highly likely to have their ventricular catheter tips surrounded by brain parenchyma on CT imaging. Conversely, patients in whom infusion tests confirmed a patent shunt were highly likely to have ventricular catheter tips surrounded by CSF on CT head imaging. This study shows such a reliable 'hydrodynamic' patency relationship with radiological positioning using 'in vivo' shunts.

Distal blockage and raised abdominal pressure can be found in $32 \%$ of cases. In total almost a half of symptomatic patients have shunts assessed as 'patent'- and this is probably a good approximation of unnecessary shunt revisions which can be avoided with additional infusion test- cheap, fast and low invasive.

Furthermore, this study uses a new, simple and reliable classification system to assess the position of ventricular catheter tips in CT head imaging, based on the prior hypotheses of previous works to suggest optimal catheter tip targets ${ }^{8-11}$. It is the unambiguous nature of this classification system, which has determined the effect of positioning factors on shunt patency. The only factor to significantly affect shunt patency was the type of matter immediately surrounding the catheter tip. A post hoc analysis showed that parietal shunts were more likely than frontal shunts to be blocked on infusion test, but this effect was due to the parietal shunts being less likely to be completely surrounded by CSF. This may be due to the anatomy of the body and trigone of the lateral ventricles in having smaller dimensions than the frontal horns and also containing choroid plexus, which has been implicated in causing shunt blockage. The suggestions for 'optimal' tip location to be anterior to the foramen of Monro in the ipsilateral frontal horn of the lateral ventricle ${ }^{8-11}$ are supported by the findings of this study. All catheter tips in this optimal position that were completely surrounded by CSF were found to be patent on infusion test; 40/40 (100\%) of optimally positioned catheters were completely surrounded by CSF but only 52/266 (21\%) of sub-optimally positioned were completely surrounded by CSF.

Only 57 infusion tests had results of hydrodynamic evidence of blockage; $19 \%$ of the whole group. We speculate that without this objective assessment of patency, a larger percentage of the group may have undergone exploratory/revision surgery on the basis of clinical presentation and CT findings. 
Also of note, of the 249 infusion tests with results of hydrodynamic patency, 29 had their catheter tips surrounded by parenchyma (12\%). It is plausible that these cases may be draining peri-ventricular oedema or extracellular/interstitial fluid hence their 'patency', but in the context of the other results of the study we suspect such catheters to be of very high risk of future blockage from the solid matter surrounding the catheter tips. From this reason, although the most important anatomical factor for shunt patency is the catheter tip being completely surrounded by CSF, it is useful to perform additional infusion test. The results of the test (if it is performed correctly), sometimes show 'functional' state of the shunt system, which may differ from 'anatomical' arrangements.

$12 \%$ of the patients had patent catheter with hydrodynamic tests but the tips of the catheter were surrounded by parenchyma. In our opinion, although it is not included in a subject of the study, those patients are of increased obstruction in a future and needs to be closely observed for potential onset of clinical symptoms.

There is an interesting result in Table 1 that it is much more common with collapsed ventricles in "Blocked shunts" group (31\% vs $12 \%$ ). In such cases first is probably overdrainage, which causes ventricles to collapse and close the ventricular inlet.

\section{Clinical context}

The results of this study support the previous arguments ${ }^{8-11}$ of ensuring optimal ventricular catheter tip positioning. We propose that the success of shunt patency in these optimal locations is due to the higher likelihood, anatomically, of the catheter tip being completely surrounded by CSF. Hayhurst et al ${ }^{15}$ demonstrated the efficacy of image guidance in ensuring such optimal positioning and in view of the inherent difficulty in blind insertion of ventricular catheters based on surface anatomical landmarks alone, this work supports such methods to ensure CSF surrounds the catheter tip of a potentially life-long implanted device. Our results support the suggestion of a recent report by Yamada et $\mathrm{al}^{27}$ to consider early revision surgery for catheter tips found to be in poor positions such as intraparenchymal or along a ventricular wall. Our results also support the usage of medium/high pressure shunt valves or judiciously lowering the pressure setting on programmable valves in order 
to avoid rapid collapse of the CSF space causing the ventricular walls to surround the catheter tip instead of CSF.

Our results specifically validate the usage of infusion studies in routine clinical practice as a reliable, low risk investigation to assess shunt dysfunction. Its usefulness for assessment shunt patency has been also confirmed by others ${ }^{18,19}$. Infusion study is a practical tool not only for testing a shunt functioning in vivo ${ }^{16,17,25}$ but also for evaluation shunt hydrodynamic performance in laboratory settings ${ }^{21,28,29}$. The latter can serve as a comparative pattern for in vivo study.

Our data show a very strong statistical correlation between the results of the infusion test and the radiological catheter position on $\mathrm{CT}$; a routinely used investigation in neurosurgical units for assessing patients with symptoms of shunt dysfunction. Not only do infusion studies provide a 'hydrodynamic' real time in-vivo assessment of shunt patency to aid the clinician in decision-making, in cases where other aspects of assessment are compromised their value is even more marked. For example in paediatric patients, patients with learning difficulties, specific neurological or psychiatric co-morbidities and patients with severe traumatic brain injury where a direct history of symptoms is not feasible or in cases where the ventricles do not alter dimensions on CT imaging in response to raised CSF pressure. Additionally, an infusion test confirming shunt patency can be a useful reassurance from clinicians to patients who may equate headaches to shunt blockages requiring revision surgery.

\section{Limitations}

In this study we compared location of ventricular catheter tip versus results of infusion study dichotomised as ventricular catheter blocked or patent. We did not consider distal blockage (valve itself or distal end) because for these cases imaging classification is not relevant.

We excluded patients with grossly abnormal intracranial anatomy, cranioplasties, decompressive craniectomy defects and with multiple shunt catheters. Hence the conclusions and recommendations of this study cannot be easily generalised to those patient groups. We also excluded patients whose scans and infusion tests were greater than three months apart. This was to help ensure that the same catheters were being 
assessed radiologically and hydrodynamically. Assessment of ventricular size in itself was not undertaken as this patient group was more ambiguous in the diagnosis of shunt dysfunction and there was not always 'baseline' imaging to compare against as many of the patients have been referred after their primary shunt surgery in other centres. This has also meant that data for interval from shunt insertion to infusion test and full etiological clinical picture at shunt insertion was not always available and hence not included in analysis, which may also be a source of bias.

Additionally, some patients underwent multiple CT scans and infusion studies due to repeated presentations with symptoms of shunt dysfunction, the data from these cases may also be a source of bias.

Occlusion of the proximal catheter often results from debris from the choroid plexus. The blockage by choroid plexus tissue and what is the expected CT images that should be seen, is not analysed as it is not possible to be determined.

We reduced measurement bias by blinding researchers to the shunt status when assessing tip position on imaging and by using an objective classification system demonstrated to not have significant inter-observer variability.

The design of the study has specifically not incorporated the intra-operative findings of those patients who went on to shunt revision. We realise that this is often the gold standard check of shunt patency however due to the limitations inherent with such data, for example lack of standardisation between intra-operative findings, missing data and uncontrolled intra-operative factors such as variable carbon dioxide partial pressure concentration affecting intracranial pressure, it was deemed that such analysis would be more confounding. Hence analysis was restricted to the imaging of catheter position and the hydrodynamic infusion test alone. However, our previous study performed on smaller simple size demonstrated high level of intraoperatively shunt blockage confirmation $(90 \%)^{16}$.

Aside from the clinical recommendations above, the methods in this study have advantages for being re-applied in future clinical shunt research. A meta-analysis of previous studies relied on the decision for surgical revision of a shunt as the surrogate 
for shunt dysfunction without confirming in all cases whether intra-operative findings were concordant with the decision ${ }^{1}$; this may not be as robust as patency/blockage demonstrated by infusion test which may be a better surrogate in future research. Other classification systems have been used to categorise proximal catheter tip position $^{10,11,15,27}$; our system has been developed from the anatomical concepts of these previous systems. However, our system is clear, easy to apply, exhaustive in describing all possible catheter tip positions, leaves little room for ambiguity in analysis and has statistical reliability to demonstrate inter-observer agreement. Both paradigms for radiological and physiological assessment of shunts in vivo may prove useful for future research.

\section{Conclusion}

Infusion tests provide a reliable assessment of proximal shunt patency in vivo as correlated with the position of the ventricular catheter on CT imaging. The most important anatomical factor is the catheter tip being completely surrounded by CSF.

\section{Conflict of Interest and Sources of Funding}

$\mathrm{MC}$ has financial interest in licensing fee of ICM+ software (Cambridge Enterprise Ltd) used for recording and analysis of infusion tests. Other authors have nothing to disclose. No funding source.

\section{Acknowledgements}

The authors gratefully acknowledge the staff of the Department of Clinical Neuroscience, Division of Neurosurgery, University of Cambridge, UK for their cooperation. MC is on unpaid leave from Warsaw University of Technology.

\section{References}

1. Stein SC, Guo W. Have we made progress in preventing shunt failure? A critical analysis. J Neurosurg Pediatr 2008;1:40-7.

2. Patwardhan RV, Nanda A. Implanted ventricular shunts in the United States: the billion-dollar-a-year cost of hydrocephalus treatment. Neurosurgery 2005;56:13944; discussion 44-5.

3. Korinek AM, Fulla-Oller L, Boch AL, Golmard JL, Hadiji B, Puybasset L. Morbidity of ventricular cerebrospinal fluid shunt surgery in adults: an 8-year study. Neurosurgery 2011;68:985-94; discussion 94-5. 
4. Reddy GK, Bollam P, Caldito G. Long-term outcomes of ventriculoperitoneal shunt surgery in patients with hydrocephalus. World Neurosurg 2014;81:404-10.

5. Sainte-Rose C, Piatt JH, Renier D, et al. Mechanical complications in shunts. Pediatr Neurosurg 1991;17:2-9.

6. Collins P, Hockley AD, Woollam DH. Surface ultrastructure of tissues occluding ventricular catheters. J Neurosurg 1978;48:609-13.

7. Sekhar LN, Moossy J, Guthkelch AN. Malfunctioning ventriculoperitoneal shunts. Clinical and pathological features. J Neurosurg 1982;56:411-6.

8. Hoffman HJ, Smith MS. The use of shunting devices for cerebrospinal fluid in Canada. Can J Neurol Sci 1986;13:81-7.

9. Becker DP, Nulsen FE. Control of hydrocephalus by valve-regulated venous shunt: avoidance of complications in prolonged shunt maintenance. J Neurosurg 1968;28:215-26.

10. Illingworth RD, Logue V, Symon L, Uemura K. The ventriculocaval shunt in the treatment of adult hydrocephalus. Results and complications in 101 patients. J Neurosurg 1971;35:681-5.

11. Albright AL, Haines SJ, Taylor FH. Function of parietal and frontal shunts in childhood hydrocephalus. J Neurosurg 1988;69:883-6.

12. Janson CG, Romanova LG, Rudser KD, Haines SJ. Improvement in clinical outcomes following optimal targeting of brain ventricular catheters with intraoperative imaging. J Neurosurg 2014;120:684-96.

13. Toma AK, Camp S, Watkins LD, Grieve J, Kitchen ND. External ventricular drain insertion accuracy: is there a need for change in practice? Neurosurgery 2009;65:1197-200; discussion 200-1.

14. Huyette DR, Turnbow BJ, Kaufman C, Vaslow DF, Whiting BB, Oh MY. Accuracy of the freehand pass technique for ventriculostomy catheter placement: retrospective assessment using computed tomography scans. J Neurosurg 2008;108:88-91.

15. Hayhurst C, Beems T, Jenkinson MD, et al. Effect of electromagneticnavigated shunt placement on failure rates: a prospective multicenter study. $\mathrm{J}$ Neurosurg 2010;113:1273-8.

16. Petrella G, Czosnyka M, Smielewski P, et al. In vivo assessment of hydrocephalus shunt. Acta Neurol Scand 2009;120:317-23.

17. Czosnyka M, Whitehouse H, Smielewski P, Simac S, Pickard JD. Testing of cerebrospinal compensatory reserve in shunted and non-shunted patients: a guide to interpretation based on an observational study. J Neurol Neurosurg Psychiatry 1996;60:549-58.

18. Eklund A, Lundkvist B, Koskinen LO, Malm J. Infusion technique can be used to distinguish between dysfunction of a hydrocephalus shunt system and a progressive dementia. Med Biol Eng Comput 2004;42:644-9.

19. Malm J, Lundkvist B, Eklund A, Koskinen LO, Kristensen B. CSF outflow resistance as predictor of shunt function. A long-term study. Acta Neurol Scand 2004;110:154-60.

20. Scollato A, Gallina P, Di Lorenzo N. Cerebrospinal fluid diversion in patients with enlarged Virchow-Robin spaces without ventriculomegaly. Acta Neurol Scand 2016;133:75-80.

21. Czosnyka ZH, Czosnyka M, Pickard JD. Shunt testing in-vivo: a method based on the data from the UK shunt evaluation laboratory. Acta Neurochir Suppl 2002;81:27-30. 
22. Katzman R, Hussey F. A simple constant-infusion manometric test for measurement of CSF absorption. I. Rationale and method. Neurology 1970;20:53444.

23. Swallow DM, Fellner N, Varsos GV, et al. Repeatability of cerebrospinal fluid constant rate infusion study. Acta Neurol Scand 2014;130:131-8.

24. Smielewski P, Czosnyka Z, Kasprowicz M, Pickard JD, Czosnyka M. ICM+: a versatile software for assessment of CSF dynamics. Acta Neurochir Suppl 2012;114:75-9.

25. Petrella G, Czosnyka M, Keong N, Pickard JD, Czosnyka Z. How does CSF dynamics change after shunting? Acta Neurol Scand 2008;118:182-8.

26. Tuli S, O'Hayon B, Drake J, Clarke M, Kestle J. Change in ventricular size and effect of ventricular catheter placement in pediatric patients with shunted hydrocephalus. Neurosurgery 1999;45:1329-33; discussion 33-5.

27. Yamada SM, Kitagawa R, Teramoto A. Relationship of the location of the ventricular catheter tip and function of the ventriculoperitoneal shunt. J Clin Neurosci 2013;20:99-101.

28. Taylor R, Czosnyka Z, Czosnyka M, Pickard JD. A laboratory model of testing shunt performance after implantation. Br J Neurosurg 2002;16:30-5.

29. Czosnyka Z, Pickard JD, Czosnyka M. Hydrodynamic properties of the Certas hydrocephalus shunt. J Neurosurg Pediatr 2013;11:198-204. 
Table 1. Results of CT scan analysis of shunt tip characteristics grouped by patency

\begin{tabular}{|c|c|c|c|c|c|}
\hline \multirow{2}{*}{\multicolumn{2}{|c|}{ CT analysis of shunt tips }} & \multicolumn{2}{|c|}{$\begin{array}{l}\text { Proximally blocked shunts as } \\
\text { confirmed by infusion tests } \\
\qquad(\mathrm{n}=57)\end{array}$} & \multicolumn{2}{|c|}{$\begin{array}{l}\text { Proximally patent shunts as } \\
\text { confirmed by infusion tests } \\
\qquad(\mathrm{n}=249)\end{array}$} \\
\hline & & $\begin{array}{l}\text { Frontal entry } \\
(\mathrm{n}=37)\end{array}$ & $\begin{array}{l}\text { Parietal entry } \\
(\mathrm{n}=20)\end{array}$ & $\begin{array}{l}\text { Frontal entry } \\
(\mathrm{n}=185)\end{array}$ & $\begin{array}{l}\text { Parietal entry } \\
(\mathrm{n}=64)\end{array}$ \\
\hline \multirow{2}{*}{$\begin{array}{l}\text { Side of shunt } \\
\text { insertion }\end{array}$} & Left & 6 & 4 & 43 & 15 \\
\hline & Right & 31 & 16 & 142 & 49 \\
\hline \multirow{3}{*}{$\begin{array}{l}\text { Location of } \\
\text { shunt tip } \\
\text { relative to } \\
\text { entry }\end{array}$} & Ipsilateral & 8 & 4 & 67 & 32 \\
\hline & Midline & 12 & 8 & 77 & 18 \\
\hline & Contralateral & 17 & 8 & 41 & 14 \\
\hline \multirow{3}{*}{$\begin{array}{l}\text { Matter } \\
\text { surrounding } \\
\text { shunt tip }\end{array}$} & $\mathrm{CSF}$ & 0 & 2 & 81 & 9 \\
\hline & Parenchyma & 26 & 7 & 23 & 6 \\
\hline & Both & 11 & 11 & 81 & 49 \\
\hline \multirow{2}{*}{$\begin{array}{l}\text { State of } \\
\text { ventricles }\end{array}$} & Collapsed & 15 & 3 & 24 & 6 \\
\hline & Non collapsed & 22 & 17 & 161 & 58 \\
\hline
\end{tabular}

Table 2. Statistical effect size of CT characteristics on proximal shunt patency

\begin{tabular}{|l|c|c|c|}
\hline CT characteristic & Degrees of freedom & Chi square statistic & $p$ - value \\
\hline Matter surrounding tip (CSF/parenchyma/both) & 2 & 63.58 & $<0.0001$ \\
\hline Entry side (left/right) & 1 & 1.18 & 0.2782 \\
\hline Approach (frontal/parietal) & 1 & 2.41 & 0.1205 \\
\hline Location of tip (ipsilateral/midline/contralateral) & 1 & 1.10 & 0.5772 \\
\hline Ventricle calibre (collapsed/not collapsed) & 1 & 0.03 & 0.86 \\
\hline *Tip anterior to foramen of Monro (Yes/No) & 1 & 0.27 & 0.6034 \\
\hline *Tip going through foramen of Monro (Yes/No) & 1 & 0.72 & 0.3954 \\
\hline *Tip abutting septum pellucidum (Yes/No) & & 0.37 & 0.5408 \\
\hline
\end{tabular}

*Frontal approach shunts only with catheter tips within lateral ventricles 


\section{Figure legends}

Figure 1. Upper panel: General scheme of infusion test set-up for testing shunt performance in-vivo. Two $21 \mathrm{G}$ needles are inserted in shunt reservoir (or prechamber). Saline filled stiff manometer line connects one needle to the pressure transducer. Through the second needle, infusion is performed. Transducer sends electric signal to pressure amplifier and computer, running infusion-supporting software reads the signal from the output of amplifier (usual sampling frequency is $100 \mathrm{~Hz}$ ).

Lower panel: Typical x-time plot of pressure recorded during study. At the baseline pressure remains usually stable. When infusion is started (this case: $1.5 \mathrm{ml} / \mathrm{min}$ rate- it is indicated by vertical bar), Pressure starts to rise gradually to reach after certain time ( 12 minutes in this case) plateau level. Resistance to CSF outflow is calculated as plateau pressure minus baseline pressure, divided by infusion rate ( in this case ((32$10 \mathrm{~mm} \mathrm{Hg}) / 1.5 \mathrm{ml} / \mathrm{min}=14.6 \mathrm{mmHg} /(\mathrm{ml} / \mathrm{min})$. X-axis: time in format: date/ hours/mins.

Figure 2. Infusion test showing the waveforms for a functional shunt. ICP- mean ICP, HR- heart rate, AMP- pulse amplitude (first harmonic) of pulsatile waveform. This is a case with patent ventricular inlet. Pressure rises gradually to reach the plateau, pulse amplitude changes proportionally to mean ICP, heart rate is followed continuously. 'Critical threshold' is a pressure level, calculated for specific type of shunt (Strata, performance level 1), as infusion rate time shunt's hydrodynamic resistance plus opening pressure plus credit for abdominal pressure $(5 \mathrm{~mm} \mathrm{Hg})$. In this case plateau pressure did not exceed 'critical threshold', which indicates that the shunt is patent distally.

Figure 3. Infusion test showing the waveforms produced in proximal obstruction. Pressure in shunt prechamber is arbitrary, without pulse waveform (AMP almost zero) and erratic (non-continuous) detection of the heart rate. After start of infusion, pressure rises almost immediately (no delay, means that the compliance of the system is much lower than in Figure 2) to the value above opening level for the shunt plus abdominal pressure. During infusion, if this is possible (it is possible in shunts like a Strata or Delta - with distal membrane Siphon-Control Device) distal occlusions are performed. Pressure rises immediately to very high values and decreases immediately after release of the occlusion.

Figure 4. Upper panel: Schematic diagram of the possible positions for the ventricular catheter tip for a right frontal shunt.

Lower left: Axial CT slice showing right frontal entry ventricular catheter with tip in ipsilateral, non-collapsed ventricle, anterior to the foramen of Monro and completely surrounded by CSF

Lower right: Axial CT slice showing a right frontal entry shunt with tip in contralateral hemisphere and completely surrounded by parenchyma. The ventricles are not collapsed.

Figure 5. Bar plot of infusion test patency by matter surrounding catheter tip. Blackpercent of infusion tests indicating ventricular block. Light grey - percent of tests indicating patent or partially patent ventricular drain. 


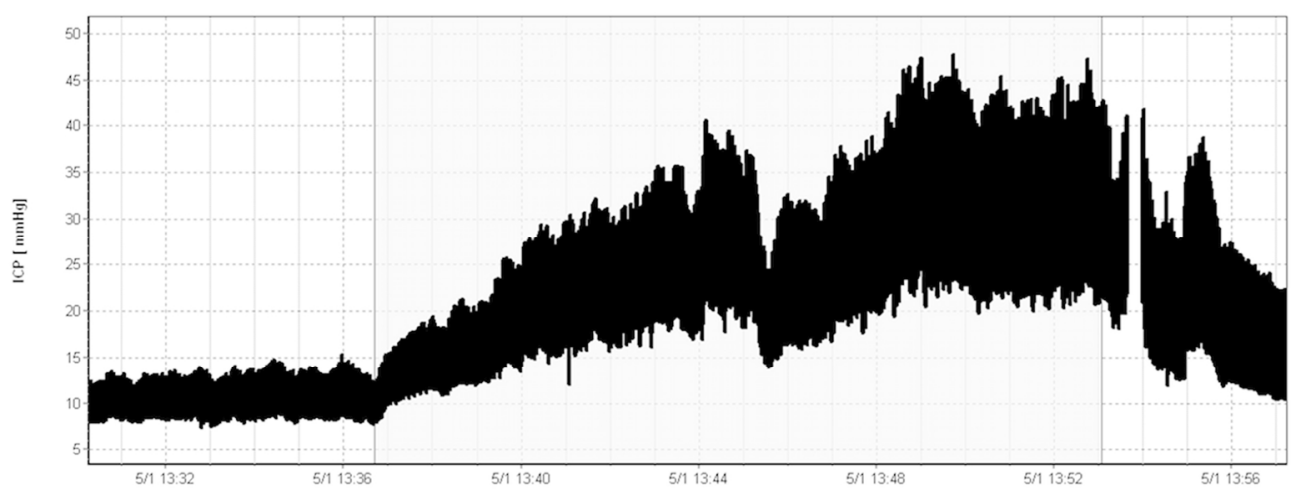

Upper panel: General scheme of infusion test set-up for testing shunt performance in-vivo. Two $21 \mathrm{G}$ needles are inserted in shunt reservoir (or pre-chamber). Saline filled stiff manometer line connects one needle to the pressure transducer. Through the second needle, infusion is performed. Transducer sends electric signal to pressure amplifier and computer, running infusion-supporting software reads the signal from the output of amplifier (usual sampling frequency is $100 \mathrm{~Hz}$ ).

Lower panel: Typical x-time plot of pressure recorded during study. At the baseline pressure remains usually stable. When infusion is started (this case: $1.5 \mathrm{ml} / \mathrm{min}$ rate- it is indicated by vertical bar), Pressure starts to rise gradually to reach after certain time ( 12 minutes in this case) plateau level. Resistance to CSF outflow is calculated as plateau pressure minus baseline pressure, divided by infusion rate ( in this case $((32-10 \mathrm{~mm}$ $\mathrm{Hg}) / 1.5 \mathrm{ml} / \mathrm{min}=14.6 \mathrm{mmHg} /(\mathrm{ml} / \mathrm{min})$. X-axis: time in format: date/ hours $/ \mathrm{mins}$.

$$
161 \times 144 \mathrm{~mm}(300 \times 300 \mathrm{DPI})
$$


Infusion test showing the waveforms for a functional shunt. ICP- mean ICP, HR- heart rate, AMP- pulse amplitude (first harmonic) of pulsatile waveform. This is a case with patent ventricular inlet. Pressure rises gradually to reach the plateau, pulse amplitude changes proportionally to mean ICP, heart rate is followed continuously. 'Critical threshold' is a pressure level, calculated for specific type of shunt (Strata, performance level 1), as infusion rate time shunt's hydrodynamic resistance plus opening pressure plus credit for abdominal pressure $(5 \mathrm{~mm} \mathrm{Hg}$ ). In this case plateau pressure did not exceed 'critical threshold', which indicates that the shunt is patent distally. $86 \times 41 \mathrm{~mm}(300 \times 300 \mathrm{DPI})$ 
Infusion test showing the waveforms produced in proximal obstruction. Pressure in shunt prechamber is arbitrary, without pulse waveform (AMP almost zero) and erratic (non-continuous) detection of the heart rate. After start of infusion, pressure rises almost immediately (no delay, means that the compliance of the system is much lower than in Figure 2) to the value above opening level for the shunt plus abdominal pressure. During infusion, if this is possible (it is possible in shunts like a Strata or Delta - with distal membrane Siphon-Control Device) distal occlusions are performed. Pressure rises immediately to very high values and decreases immediately after release of the occlusion.

\section{$93 \times 48 \mathrm{~mm}(300 \times 300$ DPI $)$}




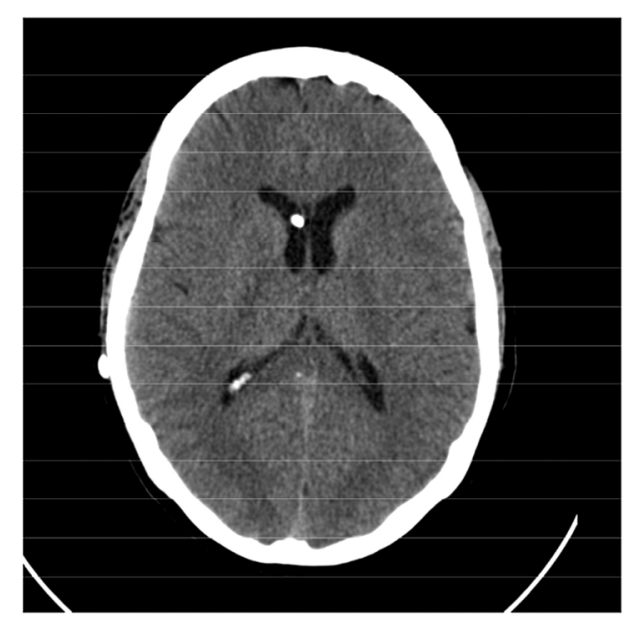

Other criteria:

Upper panel: Schematic diagram of the possible positions for the ventricular catheter tip for a right frontal shunt.

Lower left: Axial CT slice showing right frontal entry ventricular catheter with tip in ipsilateral, non-collapsed ventricle, anterior to the foramen of Monro and completely surrounded by CSF

Lower right: Axial CT slice showing a right frontal entry shunt with tip in contralateral hemisphere and completely surrounded by parenchyma. The ventricles are not collapsed.

$159 \times 141 \mathrm{~mm}(300 \times 300 \mathrm{DPI})$ 
Bar plot of infusion test patency by matter surrounding catheter tip. Black - percent of infusion tests indicating ventricular block. Light grey - percent of tests indicating patent or partially patent ventricular drain.

$75 \times 63 \mathrm{~mm}(300 \times 300$ DPI $)$ 